\title{
Assessment of patient functional performance in different knee arthroplasty designs during unconstrained squat
}

\author{
Federica Verdini ${ }^{1}$ \\ Claudio Zara 2 \\ Tommaso Leo ${ }^{1}$ \\ Alessandro Mengarelli ${ }^{1}$ \\ Stefano Cardarelli ${ }^{1}$ \\ Bernardo Innocenti ${ }^{3}$ \\ 1 Department of Information Engineering, Università \\ Politecnica delle Marche, Ancona, Italy \\ 2 Casa di Cura Stella Maris, San Benedetto \\ del Tronto, Italy \\ 3 BEAMS Department, École polytechnique de \\ Bruxelles, ULB - Université Libre de Bruxelles, \\ Bruxelles, Belgium
}

Corresponding Author:

Federica Verdini

Department of Information Engineering,

Università Politecnica delle Marche

Via Brecce Bianche 12

60131 Ancona, Italy

E-mail: f.verdini@univpm.it

\section{Summary}

Background: In this paper, squat named by Authors unconstrained because performed without constrains related to feet position, speed, knee maximum angle to be reached, was tested as motor task revealing differences in functional performance after knee arthroplasty. It involves large joints ranges of motion, does not compromise joint safety and requires accurate control strategies to maintain balance.

Methods: Motion capture techniques were used to study squat on a healthy control group (CTR) and on three groups, each characterised by a specific knee arthroplasty design: a Total Knee Arthroplasty (TKA), a Mobile Bearing and a Fixed Bearing Unicompartmental Knee Arthroplasty (respectively MBUA and FBUA). Squat was analysed during descent, maintenance and ascent phase and described by speed, angular kinematics of lower and upper body, the Center of Pressure (CoP) trajectory and muscle activation timing of quadriceps and biceps femoris.

Results: Compared to CTR, for TKA and MBUA knee maximum flexion was lower, vertical speed during descent and ascent reduced and the duration of whole movement was longer. CoP mean distance was higher for all arthroplasty groups during descent as higher was, CoP mean velocity for MBUA and TKA during ascent and descent.

Conclusions: Unconstrained squat is able to reveal differences in the functional performance among control and arthroplasty groups and between different arthroplasty designs. Considering the similarity index calculated for the variables showing statistically significance, FBUA performance appears to be closest to that of the CTR group.

Level of evidence: III a.

KEY WORDS: angular kinematics, Center of pressure, electromyography, unconstrained squat, unicompartmental knee arthroplasty, total knee arthroplasty.

\section{Introduction}

Nowadays patients with knee arthroplasty do not require only pain relief and functional restoration but also an improved and satisfactory quality of life $e^{1,2}$. In fact, an increasing number of patients want to recover a comfortable social life, including, for instance, dance and sports activities ${ }^{1}$. The current outcome evaluation procedures, such as gait analysis tests, cannot be sufficient to assess the satisfaction of these expectations. The assessment of "physical function" in patients with osteoarthritis or with Total Knee Arthroplasty (TKA) performed through the "performance-based methods" appears to be an attempt to relate the attained functional ability with user-expectation quality of life-related ${ }^{3}$. In the literature, health status questionnaires are adopted to characterise performance and provide numerical scores performance-related ${ }^{4}$. Terwee et al. ${ }^{3}$ argued that TKA patients could experience functional problems in activities more demanding than just walking and therefore the adopted scores would be inadequate to represent the physical function.

Authors of this study consider the squat a demanding task able to quantify functional performance because it involves several joints in large movements without the risk of joint damages and it requires suitable motor control strategies to maintain balance.

Both Schoenfeld et al. ${ }^{5}$ and Rossi et al. ${ }^{6}$ stressed the relevant role of squat in several activities of daily life. For Schoenfeld et al. ${ }^{5}$, this movement can be considered as a complete exercise able to enhance the 
quality of life because of the high number of muscles involved during its execution. Rossi et al. ${ }^{6}$ consider squat useful for TKA patients if performed respectively before the surgery to measure disability and after, because it enhances hip extensors and quadriceps stretching by contributing to the maintenance of balance and the control of the movement. Squat was adopted for the functional evaluation of subjects affected by patellofemoral pain syndrome ${ }^{7}$, treated with $\mathrm{TKA}^{6,8}, \mathrm{UKA}^{9}$, or after anterior cruciate ligament $(A C L)$ surgery ${ }^{10}$. In the literature, execution modality of squat was usually imposed by defining feet position (narrow or wide stance) or knee flexion angles (deep or half squat for instance) ${ }^{5,7,11,12}$. However, the constraints on the stance have relevant implications on the compressive and shear tibio-femoral forces $5,12,13$. Moreover, high amplitudes of knee flexion (greater than $90^{\circ}$ ) are considered a "common aggravating position" for the subjects, with a great effort required for "the dynamic muscular control", mainly due to the compressive forces acting on the joint ${ }^{7}$.

With respect to the above-mentioned considerations, the Authors' hypothesis was to ask the subjects to choose freely how to squat, without any constraints related to feet position, knee flexion angle, speed or upper body attitude. In fact, the Authors hypothesized that each subject felt more confident during the task if free to choose the best strategy. Authors named this kind of motor task the unconstrained squat.

Four groups were enrolled to perform the task: a healthy control group (CTR) and three groups of patients, one with a Total Knee Arthroplasty (TKA), one with a Fixed-Bearing and one with Mobile-Bearing Unicompartmental Knee Arthroplasty (respectively FBUA and MBUA).

The purpose of the present study was to assess if and how the unconstrained squat was able to reveal:

- different performances between a control population and a knee arthroplasty group;

- different performances design-related among arthroplasty groups.

In this context, performance was measured through the whole body kinematics, CoP parameters and muscular activation timing chosen to describe motor control.

\section{Materials and methods}

\section{Test population}

The study sample population consisted in:

- 7 patients with total knee arthroplasty (GKS PRIME CR-FLEX-Permedica, Italy) with PCL preserved (TKA group);

- 7 patients with mobile-bearing unicompartmental knee arthroplasty (OxfordTM III partial knee, Biomet, IN) (MBUA group);

- 8 patients with fixed-bearing unicompartmental knee arthroplasty (Accuris UNI Knee System, Smith\&Nephew, TN) (FBUA group);

- 12 healthy subjects, without symptoms of osteoarthritis who made up the control group (CTR).

Table I reports the subject' demographic data used for this study.

The same experienced surgeon (CZ) performed diagnosis of knee arthritis for all the patients and implanted the knee arthroplasty. All arthroplasties were cemented and placed through tissue saving surgical incision.

Patients had to be fully recovered from surgery: their rate, for the International Knee Society Score, had to be excellent (80-100) or good (70-79). To avoid possible effects of the arthritis at the other joints on the performance, time after surgery had to be greater than 1 year and lower than 3 .

Residual osteoarthritis and implant loosening, checked by the orthopaedic surgeon on recent radiographs of the operated limb, impairment of the contralateral knee and other joints, and/or use of a cane were considered grounds for exclusion.

The study was conducted ethically according to international standard ${ }^{14}$ and approved by the local ethics committee. Each subject signed the informed consent before beginning of the test.

\section{Unconstrained squat description}

Subjects were asked to squat at their self-pace by bending their knees up to the maximum level they felt comfortable, starting by the upright standing and then by returning to the initial position. Each subject freely chose

Table I. Anthropometrics of the sample populations. Data related to Age, Height, BMI and Time from surgery are expressed in terms of median value, Q1 and Q3.

\begin{tabular}{lllll}
\hline $\begin{array}{l}\text { Groups of } \\
\text { subjects }\end{array}$ & MBUA & FBUA & TKA & CTR \\
\hline $\mathbf{N}^{\circ}$ & 7 & 8 & 7 & 12 \\
Gender (m/f) & $2 / 5$ & $3 / 5$ & $1 / 6$ & $9 / 3$ \\
Age (year) & $68(64-72)$ & $67(61-71)$ & $70(69-71)$ & $67(63-72)$ \\
Height (m) & $1.62(1.61-1.69)$ & $1.66(1.62-1.69)$ & $1.60(1.60-1.65)$ & $1.73(1.70-1.78)$ \\
BMI (kg/m ${ }^{2}$ ) & $28(27-32)$ & $28(26-30)$ & $29(29-34)$ & $27(25-30)$ \\
$\begin{array}{l}\text { Time from surgery } \\
\text { (months) }\end{array}$ & $23(19-34)$ & $20(19-21)$ & $19(17-22)$ & \\
\hline
\end{tabular}




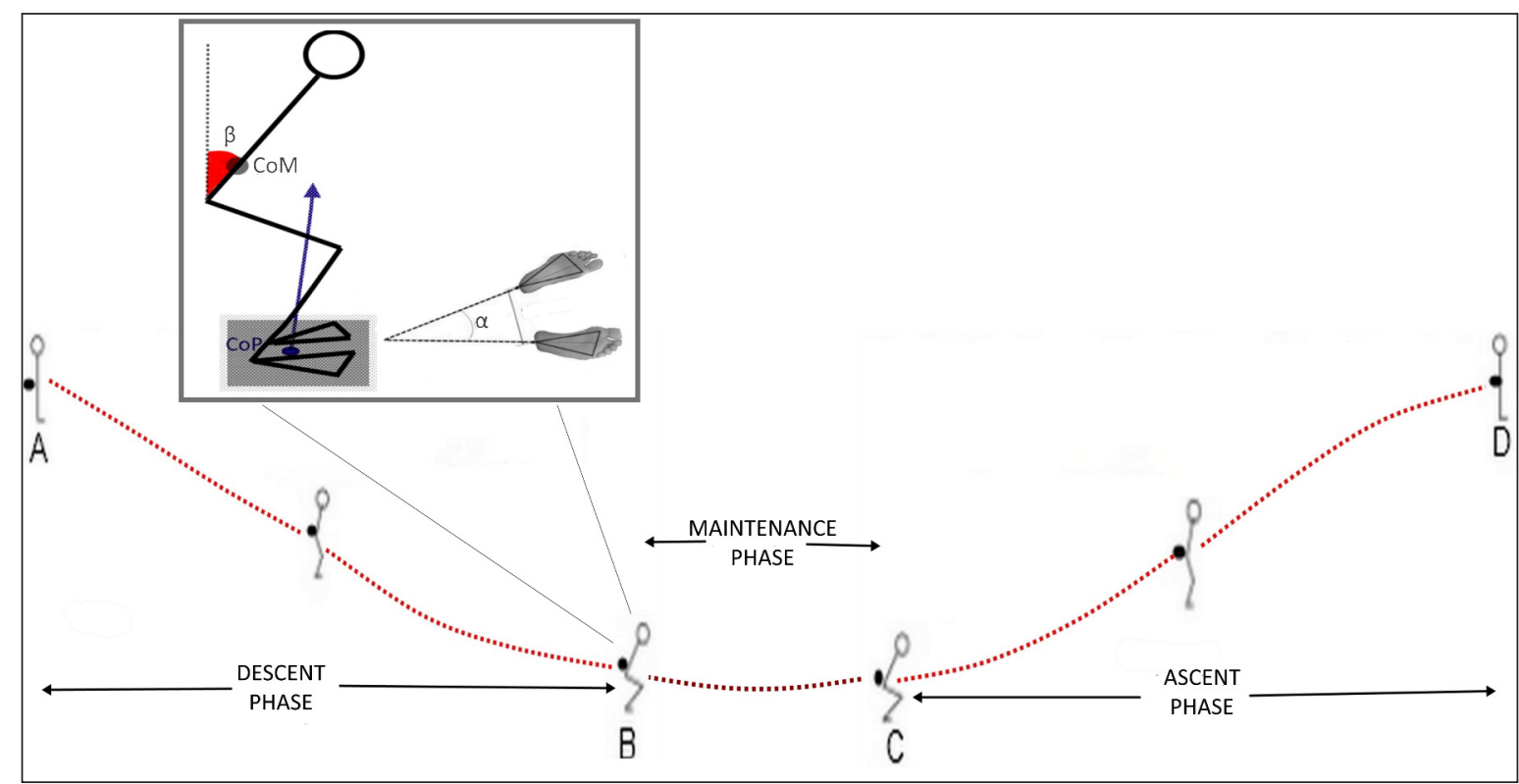

Figure 1. Kinematics parameters related to body posture during squat. The angle $\beta$, between the vertical axis and the line joining sacrum and the $7^{\text {th }}$ cervical vertebrae, defines the trunk position. The angle a between the lines through the heels and the midpoint between first and fifth metatarsal head, describes feet position. The stance width is defined as the distance between markers placed on the heels and expressed in percentage of the shoulder width (SQW\%). The dashed rectangle under the feet is the force plate that measure ground reaction forces (blu vector) and the CoP trajectory. In the bottom of the figure three phases of unconstrained squat were showed: descent, maintenance and ascent. All the phases are calculated based on the vertical position of sacrum.

the most comfortable position for the feet and arms. Each subject performed some trials to become familiar with the experimental set-up and at least three trials were analyzed and then processed.

\section{Data acquisition and processing}

For each trial, Euler angles related to ankles, knees and hips were calculated together with trunk inclination [defined, in the sagittal plane, by the angle $\beta$ between the vertical axis and the line joining sacrum (SA) and the spinous process of the $7^{\text {th }}$ cervical vertebrae (C7) (Fig. 1)]. Clusters of markers (stereophotogrammetric system ELITE, BTSEngineering, Milan, Italy, acquisition frequency $100 \mathrm{~Hz}$ ) were placed on pelvis and on thigh, shank and foot of both legs as described in ${ }^{15}$. An additional marker was placed on C7. The trunk inclination and the anterior-posterior distance between sacrum and the midpoint of the heels $\left(d_{S A-C A}\right)$ were used to obtain information about the displacement of the Center of Mass (CoM). Vertical velocity of SA was considered as a measure of subject speed during the task. It was calculated and normalised by subject's height to allow comparison among subjects.

Because each subject freely chose his/her preferred stance and feet orientation (externally or internally rotated), Authors calculated the stance width (distance between markers placed on the heels) and normalised by the shoulder width (SQW\%) $)^{10,12}$ and the angle a between feet, described by the lines through the heels and the midpoint between first and fifth metatarsal head (Fig. 1).

The displacement of SA was also used to identify three main phases of squat: in addition to descent and ascent phase, usually studied in literature, in this study was also recognised a phase, that Authors called maintenance, during which subject remained with the knees flexed, for a subject-specific time interval before the ascent.

Center of pressure (CoP) trajectory (Fig. 1) was recorded through a force plate (Bertec Corporation, Columbus $\mathrm{OH}$, acquisition frequency $500 \mathrm{~Hz}$ ). It was analyzed to calculate some descriptors of subject ability to maintain balance and to efficiently control the CoM drop and rise ${ }^{16}$. Among the parameters usually adopted in static posture analysis ${ }^{16}$, the Authors of this study will show and discuss two of them not sensitive to feet position. They are the Mean Distance (MDcop, i.e. the average distance from the mean of $\mathrm{CoP}$ ) and the Mean Velocity (MVcop, i.e. average velocity of the $\mathrm{CoP}$ ) and they are considered a measure of the effectiveness of postural control and of amount of regulatory activity respectively, needed to achieve stability ${ }^{16}$ during standing. The MDcop will increase with knee flexion and an effective motor control would allow high knee flexion without risk of falling. Therefore, MDcop will be higher for a large flexion, while high value of $\mathrm{MVcopN}$ with reduced knee flexion, will indicate a greater regulatory activity required to maintain balance. 
Surface electromyography (SEMG) of Rectus Femoris (RF), Vastus Medialis (VM) and long head of Biceps Femoris (BF) was also recorded from both legs (PocketEMG-BTS, Milan, Italy, acquisition frequency $1 \mathrm{kHz}$ ). The recommendations described in ${ }^{17}$ were followed for sensors placement and SEMG recording. The muscular activation timing was calculated through a time-frequency analysis, as described in ${ }^{18}$.

CoP parameters, linear vertical speed and muscular activation timing were calculated during descent and ascent phase separately.

\section{Statistical analysis}

Kolmogorov-Smirnov test was applied to test normality of the data distribution. Not for all the examined variables the hypothesis of normality distribution was verified so Authors decided to calculate, for each variable and for each subject, median, first and third quartiles (Q1 and Q3) values as variability measure and to adopt non-parametric methods to test significant differences.

Side-to-side comparison was performed with the use of Wilcoxon signed rank test, while Kruskal-Wallis analysis of variance test was used for inter-group comparison. Pairwise differences for each statistically significant Kruskal-Wallis test were also analysed. Differences were considered statistically significant for $\mathrm{p}<0.05$.

\section{Results}

All the subjects, comparable for age, BMI and height (Tab. I) affirmed that were able to walk without restrictions for several hours and to climb and descend stairs without the use of any support. Time after surgery was comparable among arthroplasty groups and lower than 3 years (Tab. I). Clinician present during experimental session checked the radiographic analysis to exclude possible misalignments of the arthroplasty. No case was found. Outcome and functional recovery were tested through KSS and Oxford score (Tab. II).

Maximum flexion angles for each leg were compared in each group and no statistically significant differ- ences were found. As matter of fact, the median value of each peak of flexion was bilaterally calculated and used to describe group behaviour.

Subjects adopted a narrow stance, (CTR: 76\%, TKA: 88\%; MBUA:62\%; FBUA:66\%) comparable among groups and not statistically different.

Cop mean velocity was normalised for linear vertical speed because of the recognised high correlation between both parameters (Spearman correlation $\mathrm{R}=0.7$, $\mathrm{p}<0.02$ ).

No statistically significant differences were found between groups for muscle activation timing. During the descent phase, all the arthroplasty groups started their muscular activation timing (Fig. 2) as the CTR group, at about $25 \%$ of the descent (BF), followed at $27 \%$ by the VM and finally by RF at $38 \%$. For all the arthroplasty groups, muscle remained active from the descent to the ascent phase while CTR stopped muscles activity at $90 \%$ of the descent and started at the beginning of the ascent.

During the ascent, the sequence of offset for CTR was as follows: first RF (at about $60 \%$ ), second VM (at about $78 \%$ ) and third BF (at about $88 \%$ ). This sequence was also observed for both the UKA designs, while TKA prolonged the RF activity up to $75 \%$ of the ascent duration.

\section{Arthroplasty groups versus CTR group}

The feet position was comparable for both the UKAs to CTR (a angle: FBUA:23.3, MBUA:27.1 ${ }^{\circ}$, CTR: $33.9^{\circ}$ ), while TKA group adopted a posture with feet almost parallels $(a=4.2)$, significantly different from CTR $(p=0.04)$.

Even if all subjects were able to squat without fall, the performance obtained by each group was different revealing for TKA:

- reduced knee $\left(54.4^{\circ}\right.$ w.r.t. $84.9^{\circ}$ of $C T R, p=0.03$ ) and hip $\left(41.1^{\circ}\right.$ w.r.t. $81.4^{\circ}$ of CTR, $\left.p=0.03\right)$ flexion (Fig. 3 a);

- slow normalised speed during descent (4.2 w.r.t. 19.5 of CTR, p<0.01) and ascent ( 4.5 w.r.t. 17 of CTR, , p<0.01, Fig. 3 b);

- longer maintenance (2.5s w.r.t $0.1 \mathrm{~s}$ of CTR, $\mathrm{p}<0.01$, Fig. 4 b) and descent time (2.9s w.r.t 1.7s of CTR, $p=0.0 \$$, Fig. 4 a). The prolonged duration

Table II. Results of KSS and OKS tests performed on the sample populations. For every parameter, median and $(Q 1, Q 3)$ are shown. §: significant differences between patients groups and CTR $(p<0.05)$.

\begin{tabular}{lllll}
\hline Groups of subjects & MBUA & FBUA & TKA & CTR \\
\hline $\begin{array}{l}\text { KSS, function score } \\
\text { (max 100) }\end{array}$ & $90(90-100)$ & $100(98-100)$ & $90(78-100) \S$ & $100(99-100)$ \\
$\begin{array}{l}\text { KSS, knee score } \\
\text { (max 100) }\end{array}$ & $92(80-93)$ & $90(82-93)$ & $80(78-90) \S$ & $98(96-100)$ \\
$\begin{array}{l}\text { Knee passive range } \\
\text { of flexion (degree) }\end{array}$ & $119(108-113)$ & $116(113-118)$ & $98(78-109)$ & $121(119-122)$ \\
$\begin{array}{l}\text { OKS } \\
4\end{array}$ & $41(36-45) \S$ & $44(39-48)$ & $45(32-46)$ & $47(47-48)$ \\
\hline
\end{tabular}




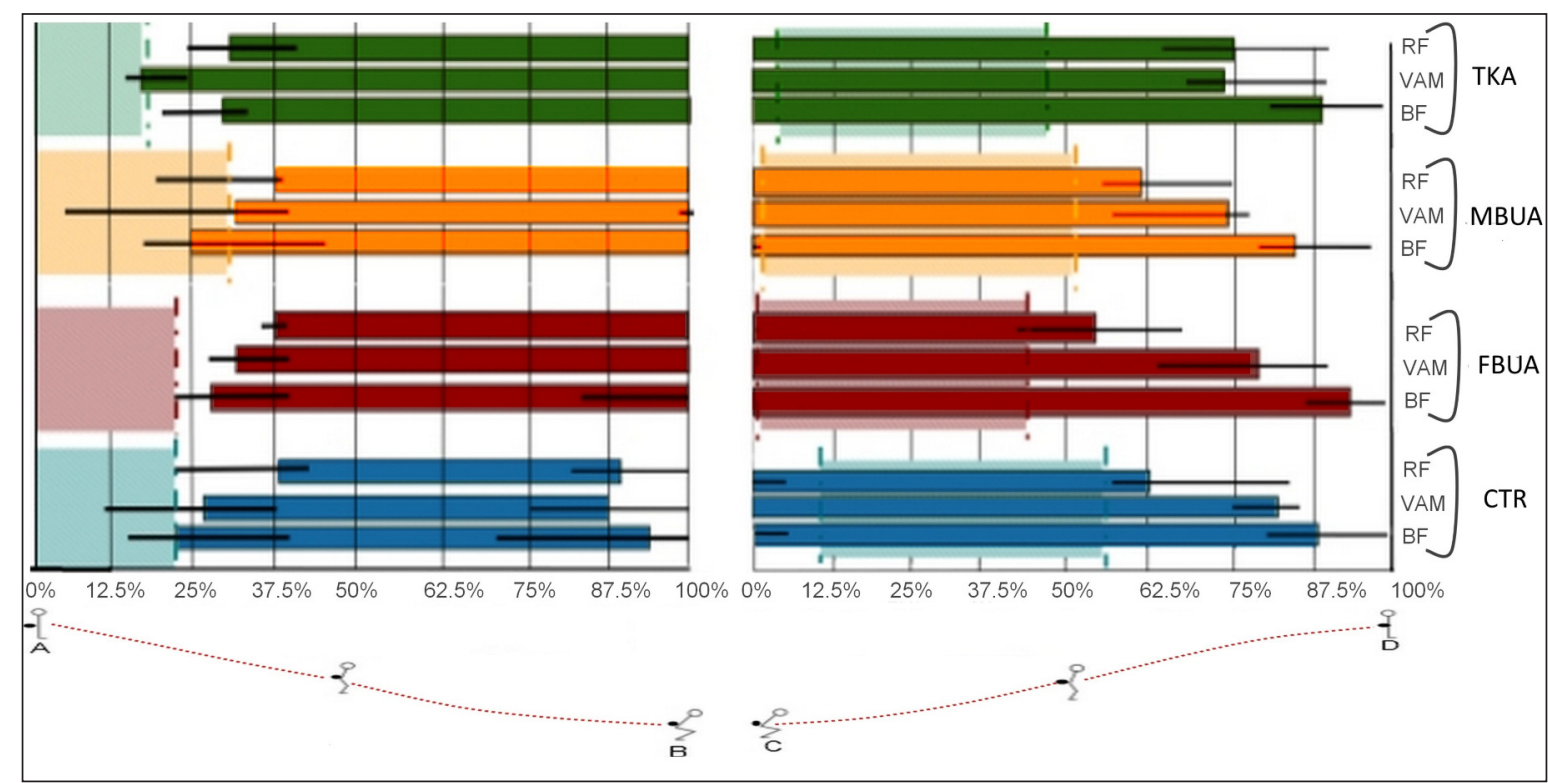

Figure 2. SEMG of rectus femoris (RF), vastus medialis (VAM) and biceps femoris (BF) for all the groups during descent phase $(A-B)$ and ascent phase (C-D). Start of each phase is labelled by $0 \%$ and the end by $100 \%$. Data are the median value of electromyographic activity of both the legs (median value and Q1, Q3 black thick lines) for each group. Dashed rectangles show duration of the downward (in descent) and the upward (in ascent) acceleration.
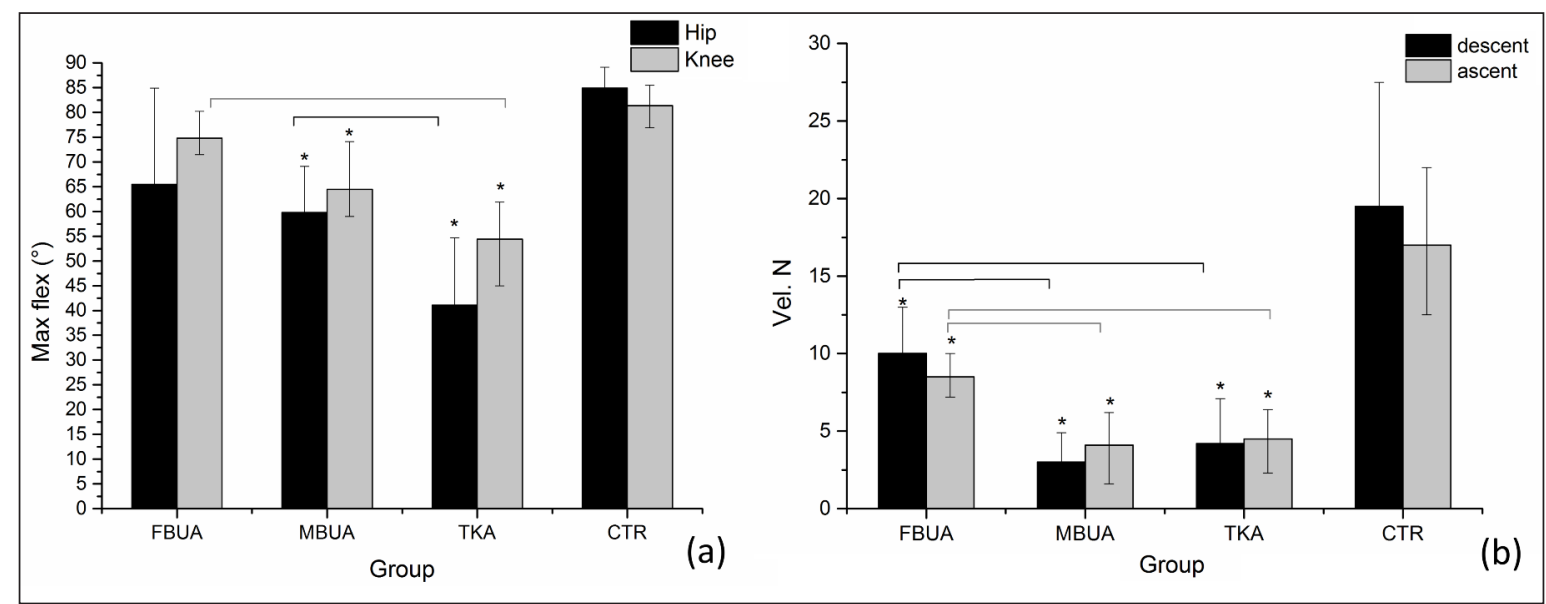

Figure 3 a, b. a) Maximum flexion of the hip (black bar) and knee (grey bar) for each group of subjects; b) Vertical speed during the descent (black bar) and the ascent (grey bar) for each group of subjects. Statistically significant comparisons $(p<0.05)$ with CTR are labelled with *, while those between arthroplasty group (Post-hoc analysis) were labelled with horizontal lines.

of the abovementioned phases produced obviously a significantly longer duration of the total movement.

The long duration of maintenance phase, associated with the prolonged muscle activity, from about $30 \%$ of descent phase, up to $75 \%$ of the ascent phase, could be due to a more cautious behaviour adopted by TKA group. For this group, this lack of confidence was also evident by trunk flexion and sacrum backward displacement, that were smaller compared to CTR (57.6 $\mathrm{mm}$ w.r.t. $128 \mathrm{~mm}$, Fig. 5). For TKA, low knee flexion was associated with high value of MDcop during the ascent (35.9 mm w.r.t. $31.2 \mathrm{~mm}$ of CTR), sign of a reduced motor control efficiency during this phase of the movement (Fig. 6 a). Although the whole movement was performed slowly, MVcopN in both the phases (19.4 and 20.1 w.r.t. 8.1 and 7.8 of CTR, $\mathrm{p}<0.01$ ) was high sign that, for this group, a greater regulatory action is required (Fig. 6 b).

With respect to CTR group, the TKA strategy appears to be efficiently represented as a "central strategy" with a reduced forward bending of the trunk and a reduced rearward displacement of the pelvis with respect to the base of support (Fig. 5). 


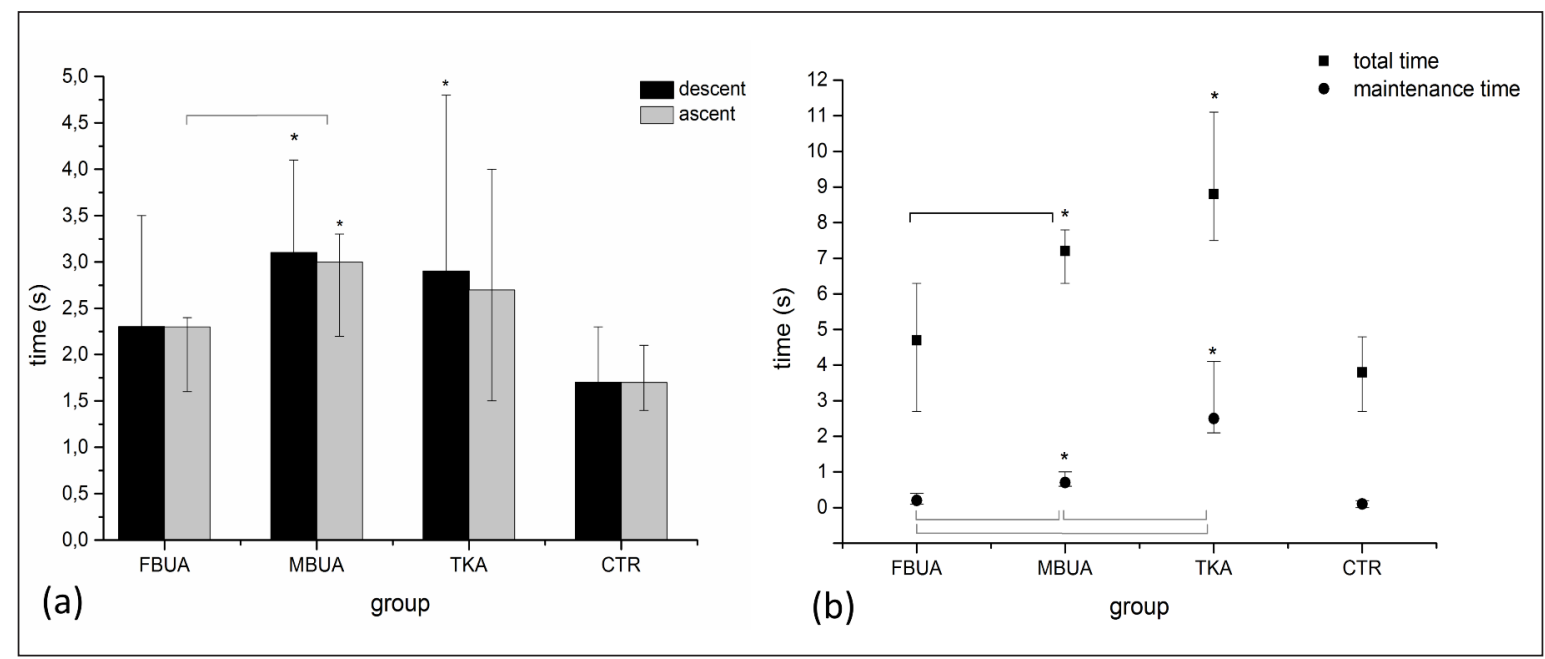

Figure 4 a, b. a) Temporal duration of the descent (black bar) and ascent phase (grey bar) for each group of subjects; b) Temporal duration of the maintenance (circle) and total phase (square). Statistically significant comparisons $(p<0.05)$ with CTR are labelled with *, while those between arthroplasty group (Post-hoc analysis) were labelled with horizontal lines.

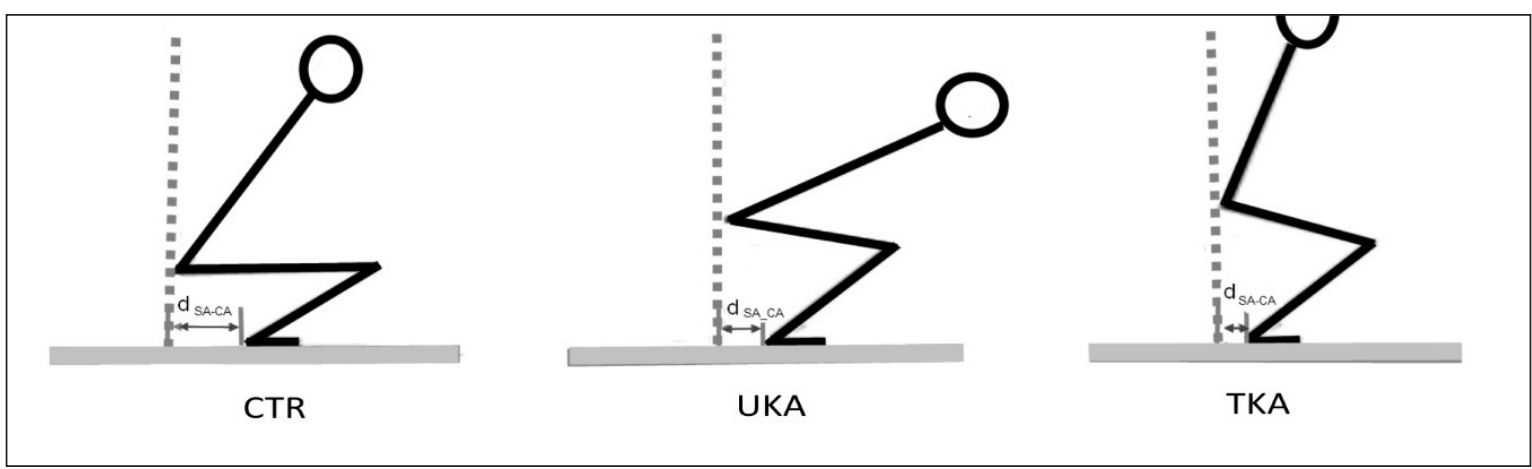

Figure 5. Modalities of execution of unconstrained squat relative to each group. Different position of the trunk and the Center of mass, as measured by dSA-CA, can be recognised.

Compared to CTR, both UKA groups seem to opt for a halfway motion strategy between that of CRT and of TKA (Fig. 5): increased trunk bending with respect to TKA and CTR and a reduced posterior displacement of sacrum with respect to CTR $(76.4 \mathrm{~mm}$ w.r.t $128 \mathrm{~mm})$.

MBUA spent a total time significantly longer than CTR (7.2 s w.r.t $3.8 \mathrm{~s} \mathrm{p<0.01)} \mathrm{to} \mathrm{complete} \mathrm{the} \mathrm{whole}$ movement and the durations of descent and ascent phase were 2 times longer with respect to the CTR $(p=0.04$, Fig. $4 a)$.

The normalised descent and ascent speeds were significantly reduced w.r.t. CTR (19.5 and 17) for both the UKAs: 10 and 8.5 for FBUA $(p<0.01), 3$ and 4 for MBUA ( $<<0.01)$ (Fig. 3 b). MBUA group showed significantly reduced hip and knee maximum flexion: $59.8^{\circ}$ for the hip and $64.5^{\circ}$ for the knee while for the control group the angles were both slightly over $80^{\circ}$. Considering the CoP parameters, MDcop was significantly reduced both in descent $(23.6 \mathrm{~mm}$ w.r.t. 30.8 $\mathrm{mm} \mathrm{p}=0.03)$ and in ascent $(24 \mathrm{~mm}$ w.r.t $31.2 \mathrm{~mm}$ for
CTR $\mathrm{p}<0.01$ ) (Fig. 6 a). The MVcopN was significantly higher $(p \leq 0.01)$ during both phases for MBUA (descent: 24.4 w.r.t. 8.1 , p $<0.01$; ascent: 16.4 w.r.t. 7.8 , $p<0.01$, Fig. 6 b) showing that, even with lower joint flexion, the regulatory action required to perform squat was high.

No statistical differences were found between FBUA e CTR in all the abovementioned parameters with the exception of linear speed both in descent (10 w.r.t. 19.5 for CTR) and in ascent phase (8.5 w.r.t. 17 for CTR, Fig. $3 \mathrm{~b}$ ) and MDcop in descent (24.2 mm w.r.t. 30.8 for CTR, p=0.03, Fig. 6 a).

To have an indication of the similarity between CTR and arthroplasty groups, Authors calculated a similarity index (SI\%) for each arthroplasty group as the mean of the percentage errors calculated for the variables showing significant differences in this study (Tab. III).

The SI\% was then defined as:

$$
S I \%_{J}=\operatorname{mean}_{i}\left(\left|\frac{\text { var }-C T R \text { var }}{C T R v a r}\right| x 100\right)
$$




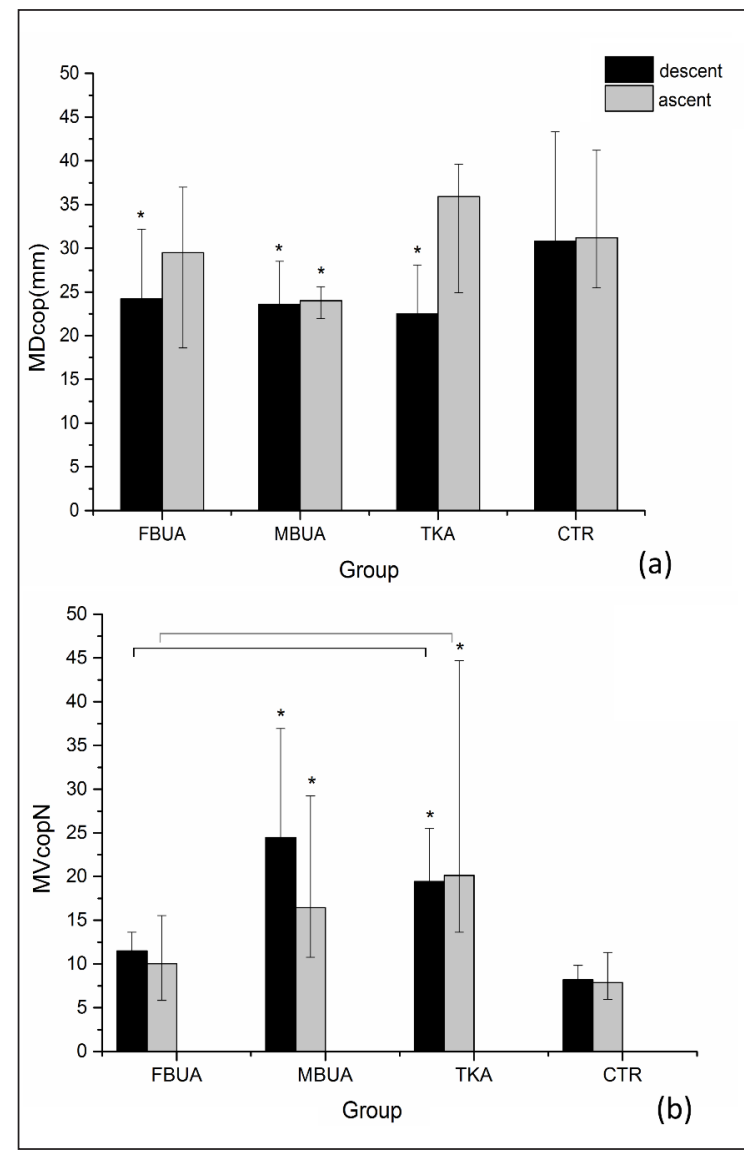

Figure 6 a, b. a) Mean distance, MDcop, parameter in mm during the descent (black bar) and ascent phase (grey bar) for each group of subjects; b) Normalised mean CoP Mean Velocity MVcopN during the descent (black bar) and the ascent phase (grey bar). Statistically significant comparisons $(p<0.05)$ with CTR are labelled with *, while those between arthroplasty group (Post-hoc analysis) were labelled with horizontal lines.

where $J=$ TKA, MBUA, FBUA; $J_{v a r}$ is the variable calculated for a given arthroplasty group and $C T R_{\text {var }}$ is the same variable calculated for the CTR group; $i$ ranged from 1 to 10 .

The lower was the SI\%, the higher was the similarity of the behaviour. Results show that a similarity can be recognisable between FBUA and CTR group while TKA group seems to be more distant from the reference behaviour.

\section{Comparison among the arthroplasty groups}

The unconstrained squat analysis performed in this study provided some parameters able to describe differences both between UKAs and TKA and between FBUA and MBUA respectively.

In particular for TKA group, maintenance time was statistically longer than MBUA (2.5s w.r.t $0.7 \mathrm{~s}$, $\mathrm{p}>0.01$ ) and FBUA (0.2s, p<0.01) (Fig. 4). Moreover TKA group lowered and raised more slowly than FBUA (speed in descent 4.2 w.r.t. 10 , in ascent 4.5 w.r.t 8.5, p $<0.01$, Fig. 3 b) reaching lower knee flexion angles $\left(54.4^{\circ}\right.$ w.r.t. $74.8^{\circ}$, Fig. 3 a). This last goal was obtained by TKA with a greater regulatory activity than FBUA as described by MVcopN both in descent $(19.4$ w.r.t. $11.5, p<0.01)$ and in ascent $(20.1$ w.r.t. 10, p<0.01, Fig. 6 b).

MBUA group spent more time than FBUA during maintenance $(0.7 \mathrm{~s}$ w.r.t. $0.2 \mathrm{~s}, \mathrm{p}<0.01)$ and ascent phase (3.0s w.r.t. 2.3s, $p=0.04$, Fig. 4). Moreover they descent $(3, p<0.01)$ and ascent $(4.1, p<0.01)$ more slowly than FBUA (10 for descent, 8.5 for ascent, Fig. 3 b).

\section{Discussion}

Nowadays, patient requirements after knee arthroplasty are focused not only on the functional restoration and on pain relief but also on the recovery of a satisfying life style ${ }^{5,19}$. As a measure of this, an increased number of patients would like to practice sports at recreational level or hobbies, such as dance. In this context, an indication of the complete functional recovery and of a satisfactory quality of life after knee arthroplasty could derive from the possibility for the patients to perform demanding task, requiring high level of coordination, in a safe and efficient manner.

In this study a demanding task as the squat, was proposed and analyzed to assess functional performance after knee arthroplasty both with respect to both a control group and arthroplasty design. Squat is generally described analyzing, for instance, descent or ascent phase, or forcing subjects to adopt a particular body attitude related to feet, trunk or knee position $5,6,11,12$. In this study, Authors asked the subjects to perform squat without constraints related to feet position, knee maximum flexion or speed of movement. Authors named this task unconstrained squat and considered descriptors of performance, parameters such as knee maximum flexion, duration of the different movement phases and two CoP parameters associated to the ability of motor control ability to maintain balance 20 .

Authors assumed that the different arthroplasty designs, each characterised by different surgical procedure, mechanical and physiological characteristics, should induce in the patients different motor behaviours. As the Authors known, no studies addressed this topic and when the knee arthritis is localised at only one knee compartment, the surgical choice is generally driven by the orthopaedic surgeon experience. Results of Bersini21, that showed as the anterior fibres of ACL become inactive after $60^{\circ}$ of knee flexion in conjunction with posterior cruciate ligament anterior bundle activation were deemed for the present study, relevant to suppose different strategies of squatting between TKA and UKA due to the different knee physiology, arthroplasty-related.

Our results showed that all subjects avoided deep squat preferring a half squat with knee flexion angles 
Table III. Similarity index calculated as the mean of the percentage errors calculated for the variables showing statistically significant differences among groups.

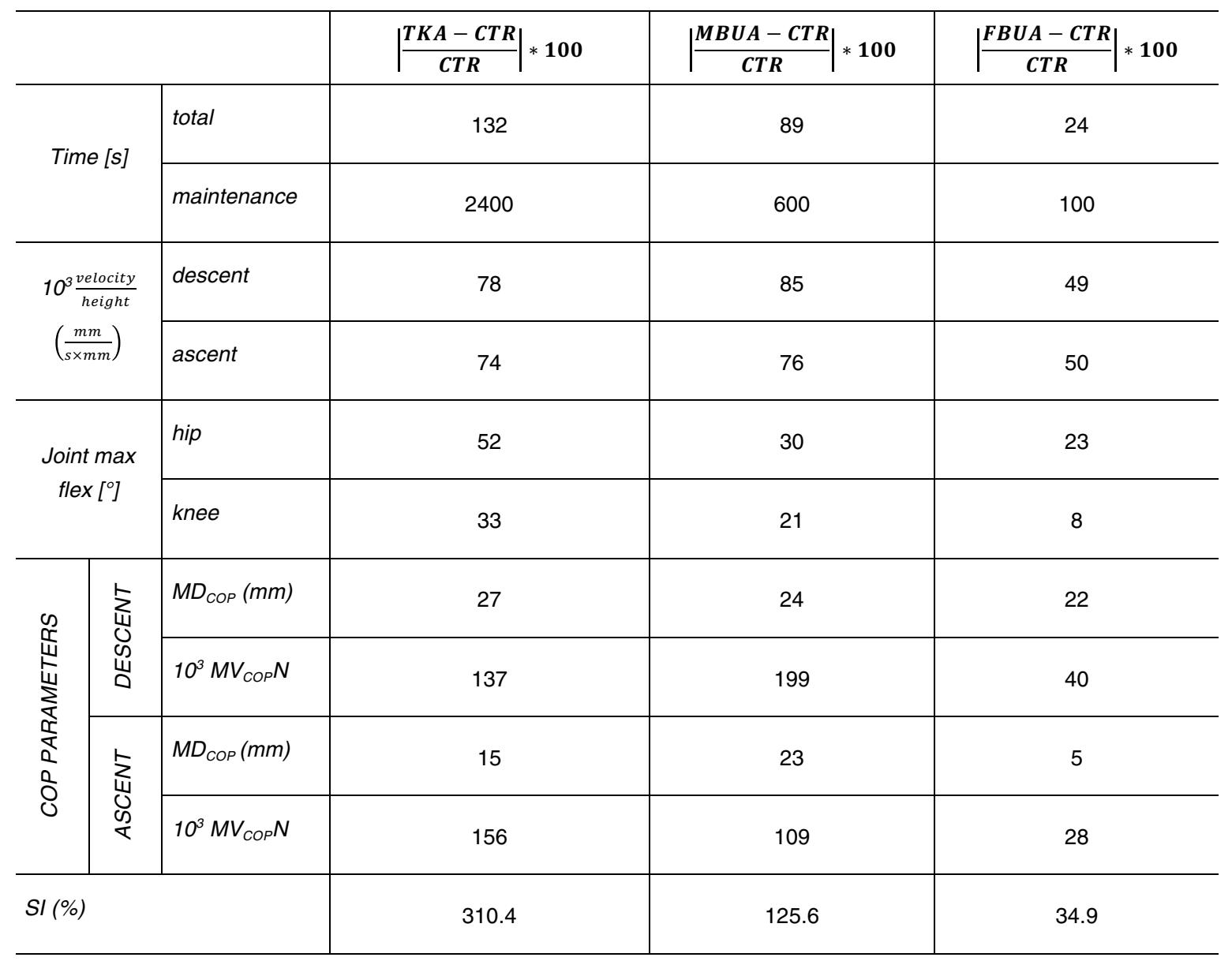

which ranged from $54.5^{\circ}$ (TKA) to 81.4 (CTR). Probably due to the closed chain nature of the exercise ${ }^{12}$, statistical differences in joints kinematics between limbs, in all the groups were not found. CoP trajectory was positioned between feet and did not reveal possible asymmetry in the body weight load. This differs from Rossi et al. ${ }^{6}$ results for which, individuals with TKA at two months after surgery, placed more body weight over the uninvolved side for three weight bearing position (standing, $30^{\circ}$ and $60^{\circ}$ knee flexion). The difference with Rossi work was probably due to the long period after surgery considered in this study, that reduced possible asymmetries observed by Rossi, just after the surgery.

Authors are not aware of studies where subjects were asked to choose the maximum knee flexion to be attained during squat. In studies, such as $6,11,12$, knee flexion was imposed and considered only with respect shear and compressive forces. The Authors' point of view in this paper is that the knee flexion is a parameter relevant to evaluate the subject level of confidence with respect to his/her functional ability in managing a demanding task. In this study, besides the descent and the ascent, often described in rela- tion to squat $6,11,20,22$, a third phase, the maintenance was identified. Its duration, particularly long for TKA group suggests that this kind of patients held the knee flexed probably to stabilize the balance before to start the ascent. Authors explained this behavior as an indication of a careful control needed to avoid loss of balance and falls. The persistent activation of knee muscles for all patients groups during this phase, is also a sign of this stabilization phase.

The muscle activity observed both during descent and ascent revealed that all the subjects tried to minimize stress to the ACL as described in ${ }^{12}$. In fact cocontractions of quadriceps and biceps femoris were present from about the $25 \%$ of the descent up to $75 \%$ of the ascent. This result and the activation sequence are confirmed in some papers ${ }^{11,12}$ and reveal that squat can be an effective exercise for the quadriceps and hamstring development.

Regardless of the prosthesis design, patients as well as CTR choose the narrow stance and a limited external rotation of the foot (a angle). This selfselected modality to perform squat, seems to be aimed to contain tibio-femoral compressive forces that increase during the drop phase and are higher 
when the knee flexion was significant (as for deep squat $)^{5,12}$ at disadvantage, probably, of shear forces ${ }^{5}$. The reduced amplitude of angle between feet $(a)$ is also a relevant parameter to infer a strategy more preservative for the knee. In fact, it was noticed ${ }^{5}$ that it should preferable to avoid large external rotations of feet because the extreme rotation of the tibia can change normal patella tracking and cause undesirable varus/valgus knee moments.

Patients involved in this study were considered clinically recovered, however, results suggest different outcomes among arthroplasty groups enrolled. Results of this study do not allow assigning a ranking of the performance because of the reduced number of subjects. However, considering the similarity index calculated with respect to the variables showing statistically significant differences, FBUA performance appears nearer to that of control group than to TKA. Kinematic parameters during the unconstrained squat, such as knee maximum flexion, speed and time during descent and ascent phase allowed to highlight differences between TKA and UKA but, overall between two UKA designs. Tasks, such as stair climbing or kneeling, proposed to differentiate the functional characteristics among different knee arthroplasty designs ${ }^{23,24}$ did not provide consistent indications about difference in performance and some previous studies deemed FBUA and MBUA designs functionally comparable 25,26 .

Cop parameters such as MDcop and MVcopN are considered in this study as descriptors of motor control effectiveness and of the regulatory activity required to maintain balance. As the Authors known this interpretation is new in the squat context. In Talarico et al. ${ }^{27}$, CoP speed was evaluated during single leg squat in single and dual task. The reduction obtained for this parameters during dual task was considered $i^{27}$ as a measure of the challenging of the dual task.

Results of this study showed, for control group, high hip and knee flexion values associated to an efficient control strategy characterised by MDcop high values. For CTR group, as a proof of the low regulatory activity required to maintain balance (efficient motor control), the low value of MVcopN is associated with high vertical speeds of CoM both in descent and in ascent phase and with a prompt change of movement direction, i.e. with a very short maintenance duration. The presence of arthroplasty modifies these strategies inducing especially those with TKA and MBUA, to adopt more cautious modality of squat.

\section{Conclusion}

In this study it was demonstrated how a simple and quite reproducible motor task as the unconstrained squat could be defined as a possible movement able to highlight differences in the performance of a patient with knee arthroplasty. Even if this is a preliminary study and further analyses are currently running in the field, this study could lead to possible improve- ment in the managing of the patient follow-up and in his rehabilitation integrating the unconstrained squat in the clinical practice of patient management. In particular, the presence of the aforementioned differences could be taken into account in the planning of the rehabilitation process, choosing different treatments also on the basis of the different physical performances highlighted in this study, particularly for TKA treated patients.

Moreover results reported in the present study could be useful in clinical contexts, supporting the surgeon in the choice of the type of arthroplasty to be implanted, due the clear inter-group differences in TKA, MBUA and FBUA subjects.

It seems worth to mention that some of such indicators, as speed, maintenance time and joint angles, can be appreciated even by visual observation of the subject during squat. Therefore, the surgeon could consider such indicators for a preliminary clinical observation and decide for a deeper analysis. A deeper study of the motor control strategies, at present simply highlighted in this paper, should be the aim of future studies also involving larger sample population. In this study, orthopaedist present during experimental session, analysed patients radiography to exclude possible recognisable anomalies. However, a measure of arthroplasty positioning was not provided even if Authors know that an arthroplasty misalignment can produce alterations on dynamics and kinematics both for TKA $8,14,28,29$ and for UKA ${ }^{30}$.

\section{References}

1. Naal FD, Fischer M, Preuss A, et al. Return to sports and recreational activity after unicompartmental knee arthroplasty. Am J Sports Med. 2007;35(10):1688-1695.

2. Mizner RL, Petterson SC, Clements KE, Zeni JA, Irrgang J, Snyder-Mackler L. Measuring Functional Improvement after Total Knee Arthroplasty Requires both Performance-Based and Patient-Report Assessments: A Longitudinal Analysis of Outcomes. J Arthroplasty. 2011;26(5):728-737.

3. Terwee CB, Mokkink LB, Steultjens MP, Dekker J. Performance-based methods for measuring the physical function of patient with osteoarthritis of hip or knee: a systematic review of measurement properties. Rheumatology (Oxf). 2006;45:890902.

4. Stratford PW, Kennedy DM, Woodhouse LJ. Performance measures provide assessments of pain and function in people with advanced osteoarthritis of the hip or knee. Phys Ther. 2006;(10)86:1489-1496.

5. Schoenfeld BJ. Squatting kinematics and kinetics and their application to exercise performance. J Strength Cond Res. 2010;24(12):3497-3506.

6. Rossi MD, Eberle T, Roche M, et al. Use of a squatting movement as a clinical marker of function after total knee arthroplasty. Am J Phys Med Rehabil. 2013;92(1):53-60.

7. Loudoun JK, Wiesner D, Goist-Foley HL, Asjes C, Loudon CL. Intra-rather reliability of functional performance tests for subjects with patellofemoral pain syndrome. J.Athletic Train. 2002;37(3):256-261.

8. Pianigiani S, Labey L, Pascale W, Innocenti B. Knee kinetics and kinematics: What are the effects of TKA misconfigurations? KSSTA Journal. 2016;24(8):2415-2421. 
9. Mochizuki T, Sato T, Tanifuji O, et al. In vivo pre- and postoperative three-dimensional knee kinematics in unicompartmental knee arthroplasty. J Orthop Sci. 2013;18:54-60.

10. Salem GJ, Salinas R. Bilateral kinematic and kinetic analysis of the squat exercise after anterior cruciate ligament reconstruction. Arch. Phys. Med. Rehabil. 2003;84:1211-1216.

11. Dionisio VC, Almeida GL, Duarte M, Hirata RP. Kinematic, kinetic and EMG patterns during downward squatting. J. of Electromyogr. Kinesiol. 2008;18(1):134-144.

12. Escamilla RF, Fleisig GS, Zheng $\mathrm{N}$, et al. Effects of techniques variations on knee biomechanics during the squat and leg press, Med. Sci Sports Exerc. 2001;33(9):1552-1566.

13. Innocenti B, Pianigiani S, Labey L, Victor J, Bellemans J. Contact forces in several TKA designs during squatting: a numerical sensitivity analysis. J Biomech. 2011;44(8):1573-1581.

14. Padulo J, Oliva F, Frizziero A, Maffulli N. Muscles, Ligaments and Tendons Journal - Basic principles and recommendations in clinical and field science research: 2016 update. MLTJ. 2016;6(1):1-5.

15. Benedetti MG, Catani F, Leardini A, Pignotti E, Giannini S. Data management in gait analysis for clinical applications. Clin. Biomech. 1998;13(3):204-215.

16. Prieto TE, Myklebust JB, Hoffmann RG, Lovett EG, Myklebust BM. Measurement of postural steadiness: differences between healthy young and elderly adults. IEEE Trans Biomed Eng. 1996;43(9):956-966.

17. Hermens HJ, Freriks B, Merletti R, et al. European recommendation for Surface ElectroMyography: results of SENIAM projects. Published by Roessingh Research and Development ISBN90-75452-15-2

18. Verdini F, Marcucci M, Benedetti MG, Leo T. Identification and characterisation of heel strike transient. Gait Posture. 2006;24 (1):77-84.

19. Weiss JM, Noble PC, Conditt MA, et al. What Functional Activities Are Important to Patients With Knee Replacements? Clinical orthopaedics and related research. 2002;404:172188.

20. Fukagawa S, Leardini A, Callewaert B, et al. Age-related changes in kinematics of the knee joint during deep squat.
Knee. 2012;19:208-212.

21. Bersini S, Sansone V, Frigo CA. A dynamic multibody model of the physiological knee to predict internal loads during movement in gravitational field. Comput Methods Biomech and Biomed Engin. 2016;19(5):571-579.

22. Kuo FC, Kao WP, Chen HI, Hong CZ. Squat-to-reach task in older and young adults: Kinematic and electromyographic analyses. Gait Posture. 2011;33:124-129.

23. Stacoff A, Kramers-de Quervain IA, Luder G, Luder G, Stussi E. Ground reaction forces on stairs-Part II: knee implant patients versus normal. Gait Posture. 2007;26:48-58.

24. Hassaballa MA, Porteous AJ, Learmonth ID, Functional outcomes after different types of knee arthroplasty: kneeling ability versus descending stairs. Med Sci Monit. 2007;13(2):7781.

25. Li MG, Yao F, Joss B, loppolo J, Nivbrant B. Wood D. Mobile vs fixed bearing unicondylar knee arthroplasty: A randomized study on short term clinical outcomes and knee kinematics. The Knee. 2006;13:365-370.

26. Catani F, Benedetti MG, Bianchi L, Marchionni V, Giannini S, Leardini A. Muscle activity around the knee and gait performance in unicompartmental knee arthroplasty patients: a comparative study on fixed and mobile-bearing designs. Knee Surg. Sports Traumatol Arthrosc. 2012;20:1042-1048.

27. Talarico MK, Lynall RC, Mauntel TC, Weinhold PS, Padua DA, Mihalik JP. Static and dynamic single leg postural control performance during dual-task paradigms, J Sports Sci. 2017;35 (11):1118-1124.

28. Pianigiani S, Chevalier Y, Labey L, Pascale V, Innocenti B. Tibio-femoral kinematics in different total knee arthroplasty designs during a loaded squat: A numerical sensitivity study. Journal of Biomechanics. 2012;45:2315-2323.

29. Innocenti B, Bellemans J, Catani F. Deviations From Optimal Alignment in TKA: Is There a Biomechanical Difference Between Femoral or Tibial Component Alignment? J Arthroplasty. 2016;31:295-301.

30. Innocenti B, Pianigiani S, Ramundo G, Thienpont E. Biomechanical effects of different varus/valgus alignments in media UKA. J. Arthroplasty. 2016;31(12):2685-2691. 